\title{
物种中文名的选定/拟定原则的建议: 以蜘蛛类为例
}

\author{
张志升 $^{1^{*}}$ 陈 建 $^{2}$ 李枢强 $^{3}$ 彭贤锦 ${ }^{4}$ 张 锋 $^{5}$ 佟艳丰 $^{6}$ 林玉成 $^{7}$ \\ 1 (西南大学生命科学学院, 三峡库区生态环境教育部重点实验室, 重庆 400715) \\ 2 (湖北大学生命科学学院, 武汉 430062) \\ 3 (中国科学院动物研究所, 北京 100101) \\ 4 (湖南师范大学生命科学学院，长沙 410006) \\ 5 (河北大学生命科学学院, 河北保定 071002) \\ 6 (沈阳师范大学化学与生命科学学院, 沈阳 110034) \\ 7 (四川大学生命科学学院, 生物资源与生态环境教育部重点实验室, 成都 610064)
}

\section{On the rules for formulating Chinese names of a species with special ref- erence to spiders}

\author{
Zhisheng Zhang ${ }^{1 *}$, Jian Chen ${ }^{2}$, Shuqiang $\mathrm{Li}^{3}$, Xianjin Peng ${ }^{4}$, Feng Zhang ${ }^{5}$, Yanfeng Tong ${ }^{6}$, Yucheng Lin ${ }^{7}$ \\ 1 Key Laboratory of Eco-environments in Three Gorges Reservoir Region (Ministry of Education), School of Life Sci- \\ ences, Southwest University, Chongqing 400715 \\ 2 College of Life Sciences, Hubei University, Wuhan 430062 \\ 3 Institute of Zoology, Chinese Academy of Sciences, Beijing 100101 \\ 4 College of Life Sciences, Hunan Normal University, Changsha 410006 \\ 5 College of Life Sciences, Hebei University, Baoding, Hebei 071002 \\ 6 College of Chemistry and Life Sciences, Shenyang Normal University, Shenyang 110034 \\ 7 Key Laboratory of Bio-resources and Eco-environment (Ministry of Education), College of Life Sciences, Sichuan Uni- \\ versity, Chengdu 610064
}

物种的学名, 即物种的科学名称 (scientific name), 是以拉丁语作为载体, 以林奈提出的双名 法来表示的。学名由拉丁化了的属名和种本名(也称 种加词)构成, 是全世界通用的名称, 为世界不同语 言国家学者之间的交流提供了重要条件。但在一些 特定语境中依然使用物种在该语言中的名称(即俗 名), 比如物种的中文名。

近年来, 大量新种和新记录种(以及科和属)相 继在中国发现。以蜘蛛类为例, 从1999年至今, 物 种数从2,361种(Song et al., 1999)猛增至4,282种(李 枢强和林玉成, 2015)。这些新增物种中, 有相当大 的一部分发表于国外期刊, 根据Web of Science数 据库统计, 2004年至今, 发表在Zootaxa期刊上的有 关中国动物新种的论文就有 1,971篇, Zookeys 期刊 上为425篇(自2008年起)。这些新种因缺乏合适的中 文名, 给国内的物种多样性及相关研究带来了一定
的困难。

因此，如何为物种选定一个合适的中文名是中 国生物多样性研究, 以及以物种为基础的生物学研 究中经常遇到的问题, 也曾有学者对此进行过讨 论, 如朱弘复等(1963)拟定夜蛾的中文名时, 提出 了“系统化”和“便于记忆和应用”两条原则; 田立新 等(1989)对昆虫的中文名拟定提出了10条建议。本 文以2015年中国蜘蛛生物多样性编目过程中遇到 的中文种名选定问题为例, 参照有关资料, 就物种 中文名的选定或拟定提出一些原则性的建议。

(1)中文名应与拉丁名一一对应, 且力求简单、 通俗易懂, 方便记忆和应用; 中文名中的种本名以 1-3个汉字为宜。如: 平腹蛛科掠蛛属的“Drassodes platnicki Song, Zhu \& Zhang, 2004”, 种本名来自世 界著名蛛形学家、美国自然历史博物馆的Norman I. Platnick的姓, 直译为“普拉蒂尼克”, 作为种本名时 
过长，因此参照中文习惯，其中文名缩短为“普氏 掠蛛”。

(2)如果该物种在某分类学文献里已记录了一 个中文名, 且无明显问题, 应尽量保留使用; 如果 该物种在多篇文献中出现了两个以上的中文名, 且 两个名称均与作者原意相近, 则应选择最先被使用 的那个。如: 栅蛛科的 “Hahnia corticicola Bösenberg \& Strand, 1906”, 最早出现的中文名为“栓栅蛛”(尹 长民和王家福，1983), 而随后又出现了另外一个中 文名“树皮栅蛛”(胡金林，1984), 种本名 “corticicola”的原意(“cortic-”意为“树皮”, “cola”意为“居住 者”)指树皮下生活的栅蛛, 而“栓”指的是树皮下的 木栓层, 暗指该蜘蛛生活于树皮下, 这两个中文名 均与作者原意相近, 但前者读写时更加简便, 因此 建议选用“栓栅蛛”。

但如果其中某个名称容易引起歧义, 则无论时 间早晚, 都应选定其他名称。

(3)物种的中文名应遵循作者发表时的原意, 或 者沿用作者提供的中文名。现代分类学文献中专门 提供了“词源学(Etymology)”, 对该种名的由来进行 了解释, 因此“译意”可以作为现代中文名的首选 项。如: 漏斗蛛科喜隙蛛属的“Himalcoelotes tortuosus Zhang \& Zhu, 2010”, 原始文献中的词源学为 "The specific epithet is derived from the Latin tortuosus, referring to the strongly coiled spermathecae.", 种本名应为“tortuosus”。本文拟定中文名为“曲喜隙 蛛”。

(4)部分物种是以国外地名命名, 或者其拉丁名 仅为字母的随意组合, 无明显含义, 此时可考虑音 译(如为地名, 考虑其本土语言译为中文名时的基 本发音, 参考《世界地名翻译大辞典》), 也可以考 虑以该种最为显著或特殊的特征为基础拟定其中 文名称。如：漏斗蛛科漏斗蛛属的“Agelena lukla Nishikawa，1980”，作者以其模式标本产地(尼泊尔 的卢卡拉镇)命名, 这里建议中文名定为“卢卡拉漏 斗蛛”。

(5)对于一些常用的拉丁语前缀或后缀, 除已经 被广泛接受的中文名外, 这里建议使用统一的翻译 原则, 尽量减少和避免不同中文名的出现。参照张 永辂(1983)的《古生物命名拉丁语》、相关词典(参 考网页http://www.thefreedictionary.com/)、维基百科 (http://en.wikipedia.org/wiki/List_of_Greek_and_Lati
n_roots_in_English)以及分类学同行的意见，将常用 的几个前、后缀总结如下:

前缀“Pseudo-"源自希腊语，意为“伪装的 (false), 假装的(pretended), 不真实的(unauthentic)”。 建议译为“伪”。如“Coelotes pseudoterrestris Schenkel, 1963”，中文名拟定为“伪地隙蛛”。

前缀 “Para-”源自希腊语, 意为“旁边的 (beside)、近似(near)”，建议译为“拟”或“副”。如 “Draconarius paratrifasciatus Wang \& Jäger, 2007”, 中文名定为“拟三分龙隙蛛”。

前缀“Sub-”意为“下面(below)”, 建议译为“亚” 或“近”。如“Orumcekia subsigillata (Wang, 2003)”, 中文名定为“亚饰扁桃蛛”; “Draconarius subabsentis Xu \& Li, 2008”, 中文名定为“近缺龙隙蛛”。

此外, 前缀“All-”建议译为“异”, 后缀“-oides” 建议译为“类”。

种以上的分类阶元选定中文名时同样可参照 上述原则。

绝大多数蜘蛛的科和属的中文名被冠以“ $\mathrm{X}$ 蛛 科”和“X蛛属”, “ $\mathrm{X}$ ”为 1 个以上的汉字, 我们建议以 1-2个汉字为宜。如属名“Himalcoelotes Wang, 2002” 译为“喜隙蛛属”; 属名“Allozelotes Yin \& Peng, 1998”的中文名选为“异狂蛛属”; Ramírez(2014)新提 升至科级地位的 “Phrurolithidae、Trachelidae、 Eutichuridae”对应的中文名分别为“刺足蛛科”、“管 蛛科”和“优列蛛科”。

但科属级名称亦有其特殊性。如异名问题: 漏 斗蛛科隙蛛亚科的“Ambanus Ovtchinnikov, 1999”被 作为“Alloclubionoides Paik, 1992”的次异名, 由于 后者与管巢蛛科的管巢蛛属(Clubiona) 有关, 而实 际上隙蛛亚科与管巢蛛亲缘关系很远, 为了不引起 歧义，我们建议保留以前为“Ambanus”选定的中文 名“满蛛属”。属名“Paracoelotes Brignoli, 1982”的中 文名曾选定为“拟隙蛛属”(张志升等, 2003), 后来发 现它是“Pireneitega Kishida, 1955”的次异名(Wang \& Jäger, 2007), 考虑到后者无特定含义, 而前者表 明该属与隙蛛属(Coelotes)具有较近的亲缘关系, 因 此我们建议沿用“拟隙蛛属”这个中文名(尹长民等, 2012)。属名“Aterigena Bolzern, Hänggi \& Burckhardt, 2010”为隅蛛属拉丁名Tegenaria所有字母的 重新组合, 无特定含义, 但因其与隅蛛属有关, 故 建议翻译为“近隅蛛属”。科级名称“Heteropodidae 
Thorell，1873”对应的中文名为“巨蟹蛛科”(朱传典, 1983; 宋大祥，1994), 后该名被作为“Sparassidae Bertkau，1872”的异名(后者被译为“遁蛛科” (王凤 振，1963)或“异足蛛科”(Fu \& Z Zhu，2008)); 由于本 科蜘蛛与蟹蛛科形态相近, 但明显比蟹蛛大, 而且 “巨蟹蛛科”这个名字远比其他两个名字的认知度 高，因此我们建议沿用“巨蟹蛛科”这个名称(李枢 强和林玉成, 2015)。

综上所述，原作者的意愿是选定或拟定物种 (及种上阶元)中文名的最根本依据。因此, 建议国内 的分类学家在发表新物种(或种上阶元)时, 尽可能 在原始文献“词源学”中标出该物种名的中文用词 (Zootaxa允许这样做), 或者在英文文章后面增加中 文摘要(Zookeys可以这样做)。据此, 国内学者选用 中文名时则有据可查, 同时也避免了翻译时选用相 同含义的不同汉字。

\section{参考文献}

Fu YN, Zhu MS (2008) A new species of the genus Pseudopoda from China (Araneae, Sparassidae). Acta Zootaxonomica Sinica (动物分类学报), 33, 657-659.

Hu JL (胡金林) (1984) The Chinese Spiders Collected from the Fields and the Forests (中国农林蜘蛛). Tianjin Science and Technology Press, Tianjin. (in Chinese)

Li SQ (李枢强), Lin YC (林玉成) (2015) Species Catalogue of China, Vol. 3 (Arachnida, Araneae) (中国生物物种名录 第三卷 蛛形纲 蜘蛛目). Science Press, Beijing. (in Chinese) (in press)

Ramírez MJ (2014) The morphology and phylogeny of dionychan spiders (Araneae: Araneomorphae). Bulletin of the American Museum of Natural History, 390, 1-374.

Song DX (宋大祥) (1994) List of Araneae families with Chinese names. Journal of Hebei Normal University (Natural Science Edition)(河北师范大学学报(自然科学版)), (Suppl.), 16-19. (in Chinese)
Song DX, Zhu MS, Chen J (1999) The Spider of China. Hebei Science and Technology Publishing House, Shijiazhuang.

Tian LX (田立新), Hu CL (胡春林) (1989) Principles and Methodologies of Insect Taxonomy (昆虫分类学的原理与 方法). Jiangsu Science and Technology Press, Nanjing. (in Chinese)

Wang FZ (王凤振) (1963) Catalogue of Chinese spiders. Journal of Jilin Medical University (吉林医科大学学报), 5, 381-456. (in Chinese)

Wang XP, Jäger P (2007) A revision of some spiders of the subfamily Coelotinae F. O. Pickard-Cambridge 1898 from China: transfers, synonymies, and new species (Arachnida, Araneae, Amaurobiidae). Senckenbergiana Biologica, 87, 23-49.

Yin CM (尹长民), Peng XJ (彭贤锦), Yan HM (颜亨梅), Bao YH (鲍幼惠), Xu X (徐湘), Tang G (唐果), Zhou QS (周青 山), Liu P (刘萍) (2012) Fauna Hunan: Araneae in Hunan, China (湖南动物志 蜘蛛类). Hunan Science and Technology Press, Changsha. (in Chinese with English abstract)

Yin CM (尹长民), Wang JF (王家福) (1983) A preliminary study on the Chinese spiders of the family Hahniidae (Arachnida: Araneida). Acta Zootaxonomica Sinica (动物分 类学报), 8, 141-145. (in Chinese with English abstract)

Zhang YL (张永辂) (1983) Palaeontological Latin in Nomenclature (古生物命名拉丁语). Science Press, Beijing. (in Chinese)

Zhang ZS (张志升), Yang ZZ (杨自忠), Zhu MS (朱明生), Song DX (宋大祥) (2003) A new species of the genus Coelotes from China, with a redescription of Coelotes modestus Simon, 1880 (Araneae: Amaurobiidae). Acta Arachnologica Sinica (蛛形学报), 12, 79-84. (in Chinese with English abstract)

Zhu CD (朱传典) (1983) A list of Chinese spiders. Journal of Bethune Medical University (白求恩医科大学学报), 9 (Suppl.), 1-130. (in Chinese)

Zhu HF (朱弘复), Chen YX (陈一心) (1963) Economic Insect Fauna of China, Vol. 3, Lepidoptera, Noctuidae (I) (中国经 济昆虫志第3卷 鳞翅目 夜蛾科 $($ 一)). Science Press, Beijing. (in Chinese) 